\title{
Non-communicable disease burden and quality of life of selected group of older people in Galle, Sri Lanka
}

\author{
Perera $\mathbf{B}^{1}$, Fernando $\mathbf{N}^{2}$, Perera $\mathbf{R}^{3}$, Wickramarachchi $\mathbf{B}^{4}$ \\ ${ }^{\prime}$ Department of Community Medicine, Faculty of Medicine, University of Ruhuna, Galle, Sri Lanka. \\ ${ }^{2}$ Department of Community Medicine, Kotalawala Defense University, Ratmalana, Sri Lanka. \\ ${ }^{3}$ Department of Psychiatry, University of Sri Jayawardenapura, Nugegoda, Sri Lanka. \\ ${ }^{1}$ Department of Nursing, Faculty of Allied Health Sciences, University of Ruhuna, Galle, Sri Lanka. \\ Correspondence: Prof. Bilesha Perera \\ email: pperera@indiana.edu \\ (B) https://orcid.org/0000-0001-5398-710X
}

\begin{abstract}
Introduction: Chronic ill health due to non-communicable diseases have already posed a great threat to the well-being of older people in Sri Lanka impairing their quality of life to a greater extent. This study was aimed to examine the impact of some chronic disease conditions on quality of life (QOL) of older people in Galle, Sri Lanka.

Methods: This cross-sectional study was conducted in 70 older people (male $=21$, female $=49$ ) aged $\geqslant 60$ years. Validated self-reported version of World Health Organization Quality of Life-BREF (WHOQOL-BREF) questionnaire which measures QOL in four dimensions; physical, psychological, environmental and social was used to assess the QOL.

Results: The age range of the participants was 60-90 years (mean $=70.3$ years, $\mathrm{SD}=6.2$ years). Of the total, 41 subjects were living with their spouse and 22 were widowed. Visual impairment (67\%), hypertension (47\%) and arthritis (34\%) were the most prevalent chronic diseases reported. QOL mean scores for all the four domains which measured in a $1-100$ scale were all higher than 65 for both men and women. Further, mean scores of QOL in all the domains in both sexes were found to be low among those who were suffering from chronic conditions, although significant differences were found in physical and psychological domains between those with and without cardiovascular diseases and hypertension $(\mathrm{p}<0.1)$.

Conclusions: QOL of older people in Galle is relatively higher compared to the QOL of community dwelling older people in many other countries. Although NCDs and other chronic conditions are prevalent in this study population, cultural and environmental factors may have contributed to improve the QOL of this population group.
\end{abstract}

Key words: Older people. NCDs, quality oflife, Sri Lanka

\section{Introduction}

The rapid aging of the population in Sri Lanka poses a challenge for well-being of its people and economic development of the country (1-3). The transition from high to low levels of birth and death rates seen in the country over the last few decades is the main reason for these observed trends in the population structure of the country. At present, the proportion of the population aged 60 years or older in the country is about $12.5 \%$, and in 2031 the figure is expected to increase to $20.7 \%(4,5)$. In 2012, old age dependency ratio was $20.1 \%$ and in 2031 the figure is expected to rise to $58.3 \%$. Non-communicable diseases (NCDs) are the main cause of deaths in older people. Conditions like Alzheimer's diseases and other cognitive impairments and disabilities are 
also prevalent in older people $(3,6)$. In 2016, 52.8\% of men and $52.1 \%$ of women aged 60 years and above were suffering from heart diseases, while $43.8 \%$ of older men and $48.6 \%$ of older women were suffering from diabetes in Sri Lanka $(7,8)$. Vision and hearing impairments are also common among older people that contributes to the morbidity giving rise to low quality of life (QOL) $(7,9)$. Social security systems and other financial benefits available for older people living today in Sri Lanka is grossly inadequate to meet expenses related to their NCD health care $(3,10)$. Thus, the burden of NCDs and other chronic health conditions in older people will become a significant challenge to health care system in the country in the near future (4-7).

These demographic transitions and trends in the prevalence of chronic diseases would eventually determine the living conditions and QOL of this group of people. Thus, one of the greatest challenges Sri Lanka has to face in the $21^{\text {st }}$ century will be to provide care for the older people with poor socioeconomic status and high prevalence of chronic comorbidities. Population dynamics and epidemiological transitions observed over the last few decades have made health care professionals and policy makers more concerned about the QOL of older people since it is directly associated with health care cost. Therefore, it is imperative to identify QOL of older people in different population segments in the country to plan and implement effective, culture sensitive and sustainable health promotion strategies to lessen health and economic burden related to aging of the population in Sri Lanka.

The World Health Organization has defined QOL as the individual's perception of their position in life in the context of the culture and the value system they inhabit, in relation to expectancies, patterns and concerns $(11,12)$. The WHOQOL-BREF is a measurement tool developed by the WHO quality of life group to measure QOL of older people (12). This tool provides scores in 4 domains of QOL; physical, psychological, environment and social. However, there are no worldwide accepted cut-off points in this tool to demonstrate better or worse QOL. The aim of this study was to study the QOL of a selected group of older people in Galle by gender and by some selected chronic diseases prevalent in this population segment.

\section{Methods}

A cross-sectional study was conducted in a total of 70 older people, aged 60 years and over recruited for the survey. The Ethics committee at the Faculty of Medicine, Galle approved the protocol of the study. Subjects were selected from two rural and to urban communities in Galle assuming that there is a difference in QOL of older people living in these two community settings. Sample size was determined considering 95\% confidence level, and expected standard deviation as $S D=14.5$ with margin of error of 5. So a minimum of 35 older people were needed for the survey. Data of a total of 70 older people were collected through face-to-face interview and analysed. Subjects of both genders, apparently healthy, independent, mobile, and who were able to communicate verbally were defined as inclusion criteria. Individuals who refused to participate in the survey due to cognitive or other communication problems were excluded.

The World Health Organization Quality of LifeBREF (WHOQOL-BREF) questionnaire that has been validated in Sri Lanka (13) was used to determine the QOL in the target group. The questionnaire consisted of 26 questions. The tool has four domains of health including physical, psychological, environmental and social. Physical health domain were evaluated through seven indicators including pain, dependence on medical aids, energy, mobility, sleep and rest, activities of daily living, and work capacity measure. Psychological health was assessed with six items including positive feeling, personal belief, concentration, bodily image, self-esteem, and negative feeling. Environmental health with eight items deals with issues related to security, physical environment and financial support, accessibility of information, leisure activity, home environment, health, and transportation. Regarding social health, three items were assessed that focused on personal relationships, social support, and sexual life. Domain scores are scaled in a positive direction; higher scores denote higher QOL. All scores are transformed to reflect a scale of 4-20. The second transformation method converts domain scores to a 0 - 100 scale. There was no overall score for the WHOQOL-BREF and each domain was calculated by summation of their specific items. Statistical Package for Social Sciences (SPSS) (version 20.0, 
Chicago, IL, USA) was used for statistical analysis of the data.

\section{Results}

A total of 70 older people ( 21 men and 49 women) were surveyed. The age range was 60 - 90 years (Mean $=70.3$ years, $S D=6.2$ years). Of the total, 41 subjects were living with spouse and 22 were widowed. Seven participants were unmarried. Two participants had never attended school and 7 had studied only up to primary education and the rest of the subjects had studied up to secondary or higher education.
The most prevalent chronic diseases reported by the participants were visual impairment $(n=47$, $67 \%)$, hypertension $(\mathrm{n}=33,47 \%)$ and arthritis $(\mathrm{n}=$ $24,34 \%)$. Of the total, 2 subjects did not report any chronic health conditions, 4 subjects reported having one chronic health condition and the rest reported having multiple co-morbidities.

Mean and standard deviation scores of the four domains of the QOL in both men and women irrespective of their health conditions are given in table 1. No gender differences were observed in the mean scores of each of the four domains of QOL investigated.

Table 1: Mean and SD of the scores of four domains of the WHOQOL-BREF in both older men and women

\begin{tabular}{llllll}
\hline Gender & & $\begin{array}{l}\text { Physical } \\
\text { Health }\end{array}$ & $\begin{array}{l}\text { Psychological } \\
\text { Health }\end{array}$ & $\begin{array}{l}\text { Environmental } \\
\text { Health }\end{array}$ & $\begin{array}{l}\text { Social } \\
\text { Relationships }\end{array}$ \\
\hline \multirow{2}{*}{ Male } & Mean & 70.4 & 74.6 & 69.2 & 65.5 \\
& SD & 13.2 & 12.1 & 10.2 & 17.5 \\
\multirow{2}{*}{ Female } & Mean & 68.5 & 72.6 & 68.8 & 68.4 \\
& SD & 14.3 & 13.6 & 13.1 & 20.6 \\
\hline
\end{tabular}

Table 2: Mean and SD of the mean scores of the four domains of the WHOQOL-BREF by disease category

\begin{tabular}{|c|c|c|c|c|c|}
\hline Disease Category & & $\begin{array}{l}\text { Physical } \\
\text { Health } \\
\text { (Mean, SD) }\end{array}$ & $\begin{array}{l}\text { Psychological } \\
\text { Health } \\
\text { ( Mean, SD) }\end{array}$ & $\begin{array}{l}\text { Environmental } \\
\text { Health } \\
\text { ( Mean, SD) }\end{array}$ & $\begin{array}{l}\text { Social } \\
\text { Health } \\
\text { ( Mean, SD) }\end{array}$ \\
\hline Diabetes & $\begin{array}{l}\text { Present }(n=15) \\
\text { Absent }(n=55)\end{array}$ & $\begin{array}{ll}65.2 & (16.6) \\
70.1 & (13.1)\end{array}$ & $\begin{array}{ll}68.3 & (15.3) \\
74.5 & (12.6)\end{array}$ & $\begin{array}{ll}65.2 & (12.4) \\
70.0 & (12.1)\end{array}$ & $\begin{array}{ll}65.0 & (16.7) \\
68.2 & (20.5)\end{array}$ \\
\hline $\begin{array}{l}\text { Cardiovascular } \\
\text { diseases }\end{array}$ & $\begin{array}{l}\text { Present }(n=13) \\
\text { Absent }(n=57)\end{array}$ & $\begin{array}{l}58.5(12.9)^{*} \\
71.5(13.1)\end{array}$ & $\begin{array}{ll}66.9 & (15.9)^{*} \\
74.6 & (12.4)\end{array}$ & $\begin{array}{ll}65.1 & (12.8) \\
69.8 & (12.1)\end{array}$ & $\begin{array}{ll}63.6 & (16.5) \\
68.4 & (20.4)\end{array}$ \\
\hline Hypertension & $\begin{array}{l}\text { Present }(n=33) \\
\text { Absent }(n=37)\end{array}$ & $\begin{array}{l}65.4(12.9)^{*} \\
72.3(14.1)\end{array}$ & $\begin{array}{ll}71.2 & (14.0) \\
75.0 & (12.6)\end{array}$ & $\begin{array}{ll}71.2 & (11.7) \\
66.9 & (12.4)\end{array}$ & $\begin{array}{ll}69.9 & (17.1) \\
65.3 & (21.7)\end{array}$ \\
\hline Asthma & $\begin{array}{l}\text { Present }(n=8) \\
\text { Absent }(n=62)\end{array}$ & $\begin{array}{lr}70.1 & (8.9) \\
68.9 & (14.4)\end{array}$ & $\begin{array}{lr}78.1 & (9.8) \\
72.6 & (13.6)\end{array}$ & $\begin{array}{lr}69.5 & (9.2) \\
68.9 & (12.5)\end{array}$ & $\begin{array}{ll}57.3 & (25.7) \\
68.8 & (18.6)\end{array}$ \\
\hline Arthritis & $\begin{array}{l}\text { Present }(n=24) \\
\text { Absent }(n=46)\end{array}$ & $\begin{array}{l}66.6(14.8) \\
70.3(13.4)\end{array}$ & $\begin{array}{ll}72.9 & (14.9) \\
73.3 & (12.5)\end{array}$ & $\begin{array}{ll}67.5 & (12.3) \\
69.7 & (12.2)\end{array}$ & $\begin{array}{ll}70.8 & (19.2) \\
65.7 & (19.9)\end{array}$ \\
\hline Visual problems & $\begin{array}{l}\text { Present }(n=47) \\
\text { Absent }(n=23)\end{array}$ & $\begin{array}{l}68.2(13.6) \\
70.9(14.4)\end{array}$ & $\begin{array}{ll}73.1 & (12.7) \\
73.3 & (14.7)\end{array}$ & $\begin{array}{ll}68.8 & (11.2) \\
69.2 & (14.2)\end{array}$ & $\begin{array}{ll}68.4 & (17.2) \\
65.5 & (24.2)\end{array}$ \\
\hline Hearing problems & $\begin{array}{l}\text { Present }(n=8) \\
\text { Absent }(n=62)\end{array}$ & $\begin{array}{l}61.1(13.8)^{*} \\
70.1(13.7)\end{array}$ & $\begin{array}{ll}74.4 & (13.4) \\
73.1 & (13.4)\end{array}$ & $\begin{array}{ll}69.9 & (15.1) \\
68.8 & (11.9)\end{array}$ & $\begin{array}{ll}73.9 & (22.4) \\
66.6 & (19.3)\end{array}$ \\
\hline
\end{tabular}

* Significant difference of the mean values were observed $(p<0.1)$ 
Over all QOL scores were seem to be low among those who were suffering from chronic conditions (table 2). However, significant differences in physical health scores were found only between those with and without cardiovascular diseases, between those with and without hypertension and between those with and without hearing problems $(p<0.1)$. A significant difference in psychological health scores were found between those with and without cardiovascular diseases $(p<0.1)$. In social health domain, however, the mean scores were slightly higher in participants with arthritis, vision and hearing problems compared to others, though no significant differences were found. In this analysis we have not analysed data separately for those with multiple chronic conditions and for those with out chronic conditions as sample sizes were too small.

\section{Discussion}

Population aging has become a vital public health issue in Sri Lanka. Many health, social and economic implications have been identified with the increasing older population in the country. There is an urgent need to understand factors related to such implications and to formulate health promotion strategies to face future public health challenges of aging population $(9,14)$. In the field of public health, QOL among older people is an important area of concern $(6,14)$. The epidemiological transition and increase in the burden of chronic conditions that cause morbidity in old age which is driven by population ageing, would eventually affect the QOL of older people. Thus, it is important to investigate the QOL experienced by older people and its associated factors so that effective health promotion strategies can be formulated and implemented to improve the QOL in this vulnerable group of people.

The QOL of older people investigated in this study was somewhat higher than that of figures found in studies conducted in many other countries. A study conducted on older people in India reported the mean scores of physical, psychological, environmental and social dimensions of QOL as $55.2(\mathrm{SD}=12.5)$, $54.6(\mathrm{SD}=11.9), 52.5(\mathrm{SD}=12.1)$ and $36.7(\mathrm{SD}=$ 16.4) respectively (16). A study conducted in Indonesia using a sample of 88 older people found that the QOL mean scores were lower than 60 for all the four domains of QOL; physical, psychological, social, and environmental (17). Sri Lankan culture which considers older people as a vital human resource in community well-being, and active life style of the majority of older people as a result of Sri Lanka being an agricultural society may have contributed to this observed higher QOL of older people in Sri Lanka $(2,3,15)$.

The effect of any chronic ill health condition was quite significant on QOL of older people. The World Health Assembly adopted the Framework Convention on Tobacco Control and the Global Strategy on Diet, Physical Activity and Health in $2004(3,6,12)$ and based on that later the 20082013 Action Plan for the Global Strategy for the Prevention and Control of Non-communicable Diseases was developed to control global burden of cardiovascular diseases, chronic respiratory diseases, and diabetes specifically prevalent in adults and older people. These chronic disease conditions make a large contribution to mortality in many low and middle-income countries across the world. NCDs are now accounted for 85 percent of the disease burden globally, and in Sri Lanka, noncommunicable diseases have become one of the leading causes of morbidity, mortality, disability and hospitalisation among the older population (3, 18).

The prevalence of visual problems (67\%), hypertension (47\%), arthritis (34\%), diabetes (21\%) and cardiovascular diseases (19\%) were comparatively higher in this target group. The figures were somewhat comparable to such figures found in other countries. For example, a study conducted in India on a sample of 300 older people found that $42.3 \%$ had hypertension, $30.7 \%$ had impaired vision and $25.3 \%$ had diabetes (16).

In our study, physical health domain of QOL was negatively affected by the presence of cardiovascular disease conditions, hypertension and hearing problems and psychological health domain was negatively affected by the presence of cardiovascular disease conditions. Other domains of QOL were not affected by chronic conditions studied in this study. However, it should be noted here that a large scale survey is needed to investigate how chronic health conditions affect QOL of older people and to confirm our results. The psychological well-being of this sample of older people could suggest a 
psychological adaptation process. A study conducted in Malaysia showed religiosity as a moderating factor in improving psychological health in older people with NCDs (19) which could also explain the better psychological health found among the subjects in this study. Social contacts and perceived social support from family members and friends are important for fulfillment of different social needs of older people. Social support has been found to be predictive of functional ability levels as well as hospital admission rates $(18,20)$. In our study social aspects of QOL seems to be satisfactory and efforts should be taken to maintain this existing conditions of social well-being in older people.

Aiming at reducing the risk of non-communicable diseases in 40-65 year olds, by detecting risk factors early and improving access to specialized care for those with a higher risk of cardiovascular disease, the Ministry of Health initiated Healthy Lifestyle Centres in 2011 (2, 3). In addition, there are other public health programs at the community level on prevention of disease and promotion of health for the elderly, such as the national Vision 2020 community programme for eye care and cataract surgery. New strategies considering the changing nature of health care and population dynamics are needed to enhance QOL of older people suffering from NCDs and other chronic ill health conditions.

In conclusion, the QOL of a group of older people in Galle, investigated in this study is praiseworthy. Although NCDs are becoming more and more prevalent in older people, QOL of this target group can be improved using available resources. Promotion of active life styles and social interactions would most probably make older people happy and content irrespective of their health conditions. Globalization, industrialization, migration and competition have created a gap between older people and the rest of the people in the society. Health and education policy makers should consider these issues in formulation of policies to bridge this gap and to promote health of older people with chronic ill health.

\section{References}

1. Samaraweera DN. Care of the elderly: a multidisciplinary approach. Journal of the Ceylon College of Physicians, 2014, 45: 45-8.
2. Samaraweera D and Maduwage S. Meeting the current and future health care needs of Sri Lanka's aging population. WHO South-East Asia Journal of Public Health, 2019; 5(2), 96-101.

3. Perera SJ. Ageing Population of Sri Lanka: Emerging issues, needs and policy implications United Nations Population Fund, Colombo, Sri Lanka, 2017.

4. De Silva WI. How serious is aging in Sri Lanka and what can be done about it? Asia-Pacific Population Journal, 1994; 9 (1), 19-36.

5. De Silva, WI. A Population Projection of Sri Lanka for the New Millennium, 2001-2101: Trends and Implications. Colombo: Institute for Health Policy, 2007.

6. Michael Engelgau, Kyoko Okamoto, Kumari Vinodhani Navaratne and Sundararajan Gopalan Prevention and Control of Selected NCDs in Sri Lanka: Policy Options and Actions, Paper prepared for World Bank, Washington, DC, USA, October 2010.

7. Department of Census and Statistics. Sri Lanka Demographic and Health Survey 2016, Ministry of Health, Nutrition and Indigenous Medicine, Colombo, Sri Lanka, September 2017.

8. Wijewardene K, Mohideen MR, Mendis S, Fernando DS, Kulathilaka T, Weerasekara D, Uluwitta P. Prevalence of Hypertension, Diabetes and Obesity: Baseline Findings of a Population-based Survey in Four Provinces in Sri Lanka. Ceylon Medical Journal, 2005; 50(2): 62-70.

9. Samaraweera D, Maduwage S. Meeting the current and future health-care needs of Sri Lanka's ageing population WHO South-East Asia Journal of Public Health, 2016; 5(2): 96-101.

10. Perera B. Social support and social security issues of elders in Sri Lanka. Galle Medical Journal, 2011;16(2): 20-3.

11. De Silva RE, Perera MSA. Quality of Life in Older Adults Attending a University Family Practice Centre in Sri Lanka. Journal of Frailty and Aging, 2018; 7(2):134-7.

12. World Health Organization Quality of Life Group. The World Health Organization Quality of Life assessment (WHOQOL): position paper from the World Health Organization. Social Science and Medicine, 1995; 41 (10):1403-9.

13. Kumarapeli K, Seneviratne R, Wijeyaratne C. Validation of WHOQOL-BREF to measure quality of life among women with polycystic ovary syndrome (PCOS). Journal of College of Community Physicians of Sri Lanka 2006; 11: $1-10$. 
14. Maduwage S. Sri Lankan 'silver-aged' population. Journal of the College of Community Physicians of Sri Lanka, 2019, 25 (1): 1-3.

15. Siddhisena KAP, Degraff DS. A Pace of Its Own: The Demography of Aging in Sri Lanka. Journal of Population Ageing 2009; 2: 77-99.

16. Ganesh Kumar S, Majumdar A, Pavithra G. Quality of Life (QOL) and Its Associated Factors Using WHOQOLBREF Among Elderly in Urban Puducherry, India Journal of Clinical and Diagnostic Research 2014; 8(1): 54-7.

17. Hidayati AR, Gondodiputro S, Rahmiati L. Elderly Profile of Quality of Life Using WHOQOL-BREF Indonesian Version: A Community-Dwelling, Althea Medical Journal, 2018;5(2):105-10.
18. Abeykoon ATPL. Aging and the health sector in Sri Lanka, Ceylon Medical Journal, 2000; 45: 52-4.

19. Sazlina SG, Zaiton A, Norafiah AZ, Hayati KS. Predictors of health related quality of life in older people with noncommunicable diseases attending three primary care clinics in Malaysia. The Journal of Nutrition Health and Aging, May 2012, DOI: 10.1007/s12603-012-0038-8.

20. Kwan P, Ali A, Deuri SP. Psychiatric morbidity, quality of life, and perceived social support among elderly population: a community-based study. Open J Psychiatry Allied Sci, 2016; 7:315. 